\title{
ZUR THEORIE DER BERÜHRUNGSTRANSFORMATIONEN DER KREISE IN EINER EBENE MIT NICHTEUKLIDISCHER MASSBESTIMMUNG.
}

\author{
Von W. Ludwig (Braunschweig).
}

Adunanza del 26 gennajo 1908 .

\section{EINLEITUNG.}

Im XXIII. Bande dieser Rendiconti habe ich ${ }^{1}$ ) auf die besondere Rolle hingewiesen, die in einer Ebene mit nichteuklidischer Massbestimmung, $\sigma$, das «absolute Polarsystem ", d.h. die durch den absoluten Kegelschnitt erzeugte polare Zuordnung, bei den Kreisverwandtschaften von $\sigma$ spielt. Der innere Grund hierfür ist darin zu suchen, dass das absolute Polarsystem selbst eine Berührungstransformation der Kreise von $\sigma$ ist, und zwar eine besonders einfache: Es ordnet ja immer zwei sich berührenden Kurven wiederum zwei sich berührende Kurven zu und vertauscht die Kegelschnitte unter einander, die den absoluten Kegelschnitt doppelt berühren, also die nichteuklidischen Kreise von $\sigma$; dabei zeichnet es sich dadurch aus, dass es den Punkten von $\sigma$ weder Punkte noch eigentliche Kreise zuordnet, sondern die in Geraden ausgearteten Kreise.

Nun lassen sich aber die Berührungstransformationen der Kreise einer Ebene $\sigma$ zurückführen auf gewisse zweideutige Transformationen der Ebenen des Raumes, die ich (E-Transformationen genannt habe ${ }^{2}$ ); also muss es auch eine (E-Transformation geben, die dem absoluten Polarsystem von $\sigma$ entspricht. Diese stellt einen Ausartungsfall der E-Transformationen dar und fügt sich nicht unmittelbar in deren allgemeine Definition ein, weshalb ich sie früher beiseite gelassen habe; aber sie ist an sich interessant wegen ihrer sehr einfachen Eigenschaften und liefert uns ausser dem absoluten Polarsystem noch eine zweite ausgezeichnete Berührungstransformation der Kreise von $\sigma$, aus der sich mit Hilfe der Spiegelungen und des absoluten Polarsystems die ganze Gruppe der Berührungstransformationen der Kreise in der nichteuklidischen Ebene $\sigma$ aufbauen lässt.

Die Aufgabe der vorliegenden Abhandlung ist es nun, diese besonders einfache

I) Über den Zusammenhang der Berührungsiransformationen der Kreise einer Ebene mit den conformen Abbildungen des Raumes (Seite 307). Diese Abhandlung wird im folgenden vorausgesetzt und soll mit $I$ bezeichnet werden.

2) Projektive Untersuchungen über die Kreisverwandtschaften der nichteuklidischen Geometrie. Habilitationsschrift. (Karlsruhe i. B. I904). Vergl. I, § I. 
⿷5-Transformation und ihre Bedeutung für die Berührungstransformationen der Kreise einer nichteuklidischen Ebene zu untersuchen.

$\int \mathrm{I}$.

\section{Die Eigenschaften der einfachen E-Transformationen.}

1. Wir nehmen im euklidischen Raume eine Kugel $\Phi$, eine Ebene $\gamma$ und ihren Pol $C$; wenn $\gamma$ von $\Phi$ in dem reellen oder imaginären Kreise $\varphi$ geschnitten wird, so wollen wir mit $\gamma$ eine nichteuklidische Ebene $\sigma$ zusammenfallen lassen, deren absoluter Kegelschnitt $\varphi$ ist, und haben dann in dem Polarsystem von $\varphi$ eine Berührungstransformation der nichteuklidischen Kreise von $\sigma$; diese projizieren wir aus $S \equiv C$ in eine Berührungstransformation der Kreise von $\Phi$ und erhalten dadurch eine Beziehung zwischen den Ebenen der Kreise von $\boldsymbol{\Phi}$, d.h. eine Transformation $\mathbb{E}^{*}$ der Ebenen des Raumes, als deren Grundkugel, Hauptebene, Centrum wir $\Phi, \gamma, C$ bezeichnen wollen. Wir erkennen sofort, dass $F^{*}$ die folgende definierende Eigenschaft hat:

Jede Transformation (5) erzeugt eine eindeutige involutorische Beziebung zwischen den Ebenenpaaren, in die die Ebenen des Raumes durch die involutorische Centralkollineation mit demselben Centrum $C$ und derselben Hauptebene $\gamma$ geordnet sind. Zu jedem solchen Ebenenpaar gehört ein Kegel II. Grades, der seine Schnittkreise mit der Grundkugel $\mathbf{\Phi}$ aus $C$ projiziert, und zwei Ebenenpaare sind einander durch $\mathfrak{E}^{*}$ zugeordnet, wenn ilre zugebörigen Kegel II. Grades in dem Bündelpolarsystem polar sind, das durch $\mathbf{\Phi}$ im Bündel $(C)$ bervorgerufen wird.

Da die Transformationen $F^{*}$ zweideutige Verwandtschaften der Ebenen des Raumes sind und in $\Phi$ Berührungstransformationen der Kreise einschneiden, fallen sie unter die Definition der E-Transformationen; sie sollen als einfache E-Transformationen bezeichnet werden; und zwar wird durch jede $\mathbb{E}^{\circ} \Phi$ so in das Ebenenbündel des Centrums $C$ von ${ }^{*}$ verwandelt, dass je zwei zugeordnete Ebenen sich auf der Hauptebene $\gamma$ schneiden, also entsprechend einer ausgearteten Centralcollineation mit den Hauptelementen $C$ und $\gamma$; daher müssen wir die Transformationen (E unter die fundamentalen \&-Transformationen rechnen. Andererseits aber ist durch die Voraussetzungen, aus denen ${ }^{3}$ ) die Eigenschaften der E-Transformationen abgeleitet worden sind, gerade der Fall ausgeschlossen, dass die mit den $₹$-Transformationen verbundenen Collineationen ausarten; wir müssen deshalb die Eigenschaften der einfachen $\mathfrak{E}$-Transformationen von neuem herleiten und können dies auch leicht auf Grund ihrer oben angegebenen Definition; dabei zeigt sich, dass die einfachen (E-Transformationen sich als besonderer Fall genau unter die fundamentalen $\mathbb{E}$-Transformationen unterordnen.

2. Als erste Eigenschaft erkennen wir:

3) Habilitationsschrift, zweiter Abschnitt, $\$ \$$ I-4. 
Jede einfache $\mathbb{E}$-Transformation $\mathfrak{F}^{*}$ ist mit ibrer inversen Transformation $\mathbb{E}^{-1}$ vollständig identisch: $\mathbb{F}^{*}=\mathfrak{E}^{*-1}$.

Je zwei einander in einer Transformation $\mathbb{E}$ entsprechende Ebenen schneiden sich auf ihrer Hauptebene $\gamma$; daraus folgt:

In einem Ebenenbïschel, dem die Hauptebene $\gamma$ einer einfachen $\mathbb{E}-$ Transformation $\mathbb{E}$. angebört, entstebt durch $\&_{*}^{*}$ eine involutorische Korrespondenz [2], die eine Involution zwischen den Ebenenpaaren der in dem Bïschel durch $C$ und $\gamma$ bestimmten Involution ist.

Jedem Ebenenbiischel, dessen Axe Tangente der Grundkugel $\Phi$ ist, sind durch E* zwei, zu ibm mit $\gamma$ als Perspektivitätsebene perspektive Ebenenbüschel zugeordnet, deren Axen ebenfalls $\boldsymbol{\Phi}$ berübren.

Auf Grund der Erzeugung der Flächen II. Grades durch projektive Ebenenbüschel lassen sich aus diesem Satze die folgenden Eigenschaften ableiten:

(5* verwandelt jede, der Grundkugel $\Phi$ umscbriebene Fläche II. Grades $X_{1}$ - und zugleich auch die Fläche $X_{2}$, die ibr in der involutorischen Centralcollineation $\mathbb{E}$ mit den nämlichen Hauptelementen gepaart ist - in zquei ebensolche Fläcben $X_{1}^{\prime}$ und $X_{2}^{\prime}$, die einander in $C^{5}$ zugeordnet sind, und die Hauptebene $\gamma$ in demselben Kegelschnitt scbneiden wie $X_{1}$ und $X_{2}$.

Dabei bestehen zwischen den Berührungsebenen von $X_{\mathrm{r}}$ und $X_{\mathrm{r}}^{\prime}$, sowie von $X_{\mathrm{r}}$ und $X_{2}^{\prime}$, sowie von $X_{2}$ und $X_{1}^{\prime}$, sowie von $X_{2}$ und $X_{2}^{\prime}$ umkehrbar eindeutige Zuordnungen derart, dass je zwei einander entsprechende Ebenen sich auf $\gamma$ schneiden, also Centralcollineationen.

Entartet $X_{1}$ in ein Ebenenbïndel $\left(P_{1}\right)$, so fallen $X_{1}^{\prime}$ und $X_{2}^{\prime}$ in eine Fläche $X^{\prime} z^{u-}$ sammen; die Ebene des Berïbrungskreises von $X^{\prime}$ und $\Phi$ verbindet das Centrum $C$ der $F^{*}$ mit der, i. Bez. auf $\Phi$ genommenen Polare der Geraden $\overline{C P_{1}}$. Liegt $P_{1}$ in der Hauptebene $\gamma$, so ist $X_{1} \equiv X_{2} \equiv X^{\prime}$; nur in diesem Falle wird $X^{\prime}$ auch ein Ebenenbïndel, [nämlich das Ebenenbïndel. $\left(P_{\mathrm{1}}\right)$ selbst].

Umgekehrt entsprechen einer Fläche II. Grades $X$ zu'ei Ebenenbündel, wenn $X$ die Grundkugel $\Phi$ längs eines Kreises berïhrt, dessen Ebene durch das Centrum $C$ von $\varpi^{*}$ läıft.

In diesem Falle ist $X$ in der involutorischen Centralcollineation 5 mit den Hauptelementen $C, \gamma$ sich selbst gepaart; es giebt aber noch andere derartige Flächen II. Grades :

Eine Fläche II. Grades X, die die Grundkugel $\boldsymbol{\Phi}$ längs ibres Scbnittkreises mit der Hauptebene $\gamma$ berübrt, gebt durch $\mathscr{F}^{\circ}$ in eine ebensolche Fläche $X^{\prime}$ ïber. Jeder Tangentialkegel von $X$, dessen Scheitel in $\gamma$ liegt, scbneidet $\Phi$ in zwei Kreisen, deren Ebenen $X^{\prime}$ berïbren, - und umgekehrt. Einmal fallen $X$ und $X^{\prime}$ zusammen in die "Hauptfläche " von $\mathbb{F}^{*}$, die durch $E^{\circ}$ so in sich selbst übergeführt wird, dass jeder sie berübrenden Ebene erstens diese selbst und zweitens die ibr in 5 gepaarte entspricht.

3. Die Aufeinanderfolge mehrerer einfachen $\mathbb{E}$-Transformationen müssen wir sorgfältig und ausführlich untersuchen, da es sich um mehrdeutige Verwandtschaften handelt; deshalb sollen hierbei auch die Beweise mitgeteilt werden. Zunächst sei $\mathfrak{F}^{*}$ eine 
einfache $\mathbb{E}$-Transformation mit den Hauptelementen $C, \gamma$ und $\mathfrak{T}$ eine die Grundlugel $\Phi$ in sich selbst überführende involutorische Centralcollineation, in der den Elementen $C, \gamma$ die Elemente $D, \delta$ entsprechen mögen. Wenden wir dann $\mathfrak{T}$ auf $\mathscr{F}^{*}$ an, d.h. bilden wir die Transformationen $\mathfrak{I} \mathbb{E} \mathfrak{T}$, so entspricht in dieser einem Ebenenpaar $n_{1}$, $n_{2}$ ein Ebenenpaar $n_{1}^{\prime}, n_{2}^{\prime}$, wenn die ihnen durch $\mathfrak{I}$ zugeordneten Ebenenpaare $\varepsilon_{1}, \varepsilon_{2}$ und $\varepsilon_{1}^{\prime}, \varepsilon_{2}^{\prime}$ zwei sich in entsprechende Ebenenpaare sind; in diesem Falle sind $n_{1}$ und $n_{2}$ sowohl, wie $n_{1}^{\prime}$ und $n_{2}^{\prime}$ durch $D$ und $\delta$ harmonisch getrennt, und die beiden Kegel II. Grades die aus $D$ die Schnittkreise von $\Phi$ mit $n_{1}$ und $n_{2}$, bezw. mit $n_{1}^{\prime}$ und $n_{2}^{\prime}$ projizieren, sind zu einander polar in dem durch $\Phi$ im Bündel $(D)$ erzeugten Bündelpolarsystem. Also ist $\mathfrak{T} \mathfrak{E}^{*} \mathfrak{T}$ ebenfalls eine einfache $\mathbb{E}$-Transformation, nämlich diejenige mit den Hauptelementen $D$ und $\delta$. - Sind ferner $\mathscr{F}_{\mathrm{i}}^{*}$ und $\mathscr{F}_{2}^{*}$ zwei einfache E. Transformationen mit derselben Grundkugel $\Phi$ und den Hauptelementen $C_{1}, \gamma_{1}$ bezw. $C_{2}, \gamma_{2}$, so kann man auf zwei Weisen eine involutorische Centralcollineation $\mathfrak{I}$ konstruieren, die $\Phi$ in sich selbst und $\gamma_{1}$ in $\gamma_{2}$, also auch $C_{1}$ in $C_{2}$ überführt; man braucht nur als Centrum von $\mathfrak{I}$ den Scheitel eines der beiden Kegel II. Grades zu nehmen, die durch die Schnittkreise von $\Phi$ mit $\gamma_{1}$ und $\gamma_{2}$ gehen. Das heisst :

Jede einfache (E-Transformation kann (auf zwei $W$ eisen) aus jeder anderen ebensolchen Transformation mit derselben Grundkugel $\Phi$ abgeleitet werden durch Anwendung einer $\mathbf{\Phi}$ in sich selbst überfübrenden involutorischen Centralcollineation.

Als besonderen Fall bemerken wir:

Ist $\mathbb{E}$ die involutorische Centralcollineation, die mit $\mathfrak{E}$ die Hauptelemente gemcinsam bat, so ist

$$
\mathfrak{E} \mathfrak{E}=\mathfrak{E} \mathfrak{E}=\mathfrak{E} \mathfrak{E}=\mathfrak{E} .
$$

4. Ferner bilden wir aus zwei einfachen $\mathbb{E}$ Transformationen $\mathscr{E}_{1}^{*}$ und $\mathbb{E}_{2}^{*}$ mit dcrselben Grundkugel $\Phi$ und den Hauptelementen $C_{1}, \gamma_{1}$ und $C_{2}, \gamma_{2}$ ihre Aufeinanderfolge, die Transformation $\mathscr{F}_{1}^{*} \mathfrak{F}_{2}^{*}: \Phi$ wird durch $\mathscr{E}_{1}^{*}$ in das Ebenenbündel $\left(C_{1}\right)$, d.h. in den als Ebenenort aufgefassten, von $C_{\mathrm{r}}$ an $\Phi$ gehenden Tangentialkegel $\Gamma_{1}$ und dieser wieder durch $\mathbb{E}_{2}$ in eine der $\Phi$ umschriebene Fläche II. Grades $\Psi$ verwandelt, die i. A. kein Kegel ist und $\gamma_{2}$ in demselben Kegelschnitt schneidet wie $\Gamma_{1}$. Die Ebene $\pi$ des Berührungskegelschnittes von $\boldsymbol{\Phi}$ und $\boldsymbol{\Psi}$ verbindet $C_{2}$ mit der i. Bez. auf $\boldsymbol{\Phi}$ genommenen Polare von $\overline{C_{1} C_{2}}$, d. h. mit der Schnittgeraden ${\overline{\gamma_{1}}}_{\mathrm{r}}$, und der Pol $P$ von $\pi$ (sowohl in Bezug auf $\Phi$ als auf $\Psi$ ) liegt in $\gamma_{2}$ und auf $\bar{C}_{1} C_{2} .-Z$ wei Berührungsebenen $\varepsilon_{\mathrm{I}}, \varepsilon_{2}$ von $\Phi$, die sich in einer Geraden $g_{1}$ von $\gamma_{\mathrm{I}}$ schneiden, gehen durch $\mathbb{E}_{1}^{*}$ über in die Ebene $\varepsilon^{\prime} \equiv\left(C_{1} g_{1}\right)$ und diese, die $\gamma_{2}$ in $g_{2}$ schneiden möge, durch $\mathbb{E}_{2}^{\prime}$ in die aus $g_{2}$ an $\Psi$ zu legenden Tangentialebenen $\varepsilon_{1}^{\prime \prime}, \varepsilon_{2}^{\prime \prime}$. Es besteht also $z$ wischen den Geradenfeldern in $\gamma_{1}$ und $\gamma_{2}$ eine Perspektivität mit $C_{1}$ als Perspektivitätscentrum; und den Tangentialebenen $\varepsilon_{1}, \varepsilon_{2}$, die aus einer Geraden $g_{1}$ von $\gamma_{1}$ an $\Phi$ gehen, sind durch $\mathscr{E}_{1}^{*} \varpi_{2}^{*}$ die Tangentialebenen $\varepsilon_{1}^{\prime \prime}, \varepsilon_{2}^{\prime \prime}$ zugeordnet, die aus der zu $g_{s}$ perspektiven Geraden $g_{2}$ von $\gamma_{2}$ an $\Psi$ gehen. Insbesondere sind in dieser Perspektivität zwischen $\gamma_{1}$ und $\gamma_{2}$ die beiden auf dem Kegel $\Gamma_{1}$ gelegenen Kegelschnitte $\left(\Phi \gamma_{1}\right)$ und $\left(\Psi \gamma_{2}\right)$ einander zugeordnet, und die Perspektivität zwischen $\gamma_{1}$ und $\gamma_{2}$ ist als Collineation, die zwei ge- 
gebene Kegelschnitte in einander überführt, durch drei, auf diesen Kegelschnitten gewählte Paare entsprechender Punkte, $L, L^{\prime \prime} ; M, M^{\prime \prime} ; N, N^{\prime \prime}$ bestimmt. - Ist $\mathfrak{S}$ eine der beiden räumlichen Centralcollineationen, die $P$ und $\pi$ zu Hauptelementen haben und $\Phi$ in $\Psi$ verwandeln, so erzengt sie in der Ebene $\gamma_{2}$, die ja durch $P$ geht, eine ebene Centralcollineation, die $P$ und $\overline{\pi \gamma_{2}} \equiv \bar{\gamma}_{1} \gamma_{2}$ zu Hauptelementen hat und den Kegelschnitt $\left(\Phi, \gamma_{2}\right)$ in den Kegelschnitt $\left(\Psi \gamma_{2}\right)$ überführt; dabei seien $L^{*}, M^{*}, N^{*}$ die Punkte von $\left(\Phi \gamma_{2}\right)$, denen die Punkte $L^{\prime \prime}, M^{\prime \prime}, N^{\prime \prime}$ von $\left(\Psi \gamma_{2}\right)$ entsprechen. Durch die Punktepaare $L, L^{*} ; M, M^{*} ; N, N^{*}$ wird zwischen den Ebenen $\gamma_{1}$ und $\gamma_{2}$ eine neue Collineation festgelegt, die den Kegelschnitt $\left(\Phi \gamma_{1}\right)$ in den Kegelschnitt $\left(\Phi \gamma_{2}\right)$ verwandelt, und zwar setzt sie sich zusammen aus der ursprünglichen Perspektivität zwischen $\gamma_{\text {t }}$ und $\gamma_{2}$ (bestimmt durch $L, L^{\prime \prime} ; M, M^{\prime \prime} ; N, N^{\prime \prime}$ ) und aus der Umkehrung der soeben in $\gamma_{2}$ betrachteten Centralcollineation (chatakterisiert durch $L^{\prime \prime}, L^{\prime} ; M^{\prime \prime}, M^{*} ; N^{\prime \prime}, N^{*}$ ); sie lässt also, wie diese beiden, alle Punkte der Geraden $\overline{\gamma_{1} \gamma_{2}}$ ungeändert, d.h. sie ist ebenfalls eine Perspektivität und hat zum Perspektivitätscentrum $T$ den auf $\overline{C_{1} C_{2}}$ liegenden Scheitel des einen der beiden Kegel II. Grades, die durch die Kegelschnitte $\left(\Phi \gamma_{1}\right)$ und $\left(\Phi \gamma_{2}\right)$ gehen. - Es giebt gerade zwei Collineationen, die $\Phi$ so in sich selbst überführen, dass den Punkten $L, M, N$ die Punkte $L^{*}, M^{*}, N^{*}$ entsprechen ${ }^{4}$ ); dies sind die involutorische Centralcollineation $\mathfrak{T}$, die $T$ als Centrum und die i. Bez. auf $\Phi$ genommene Polarebene $\tau$ von $T$ als Hauptebene hat, und die involutorische Collineation mit Axen (windschiefe Involution), die sich aus der involutorischen Centralcollineation $\mathfrak{E}_{1}$ mit den Hauptelementen $C_{1}, \gamma_{1}$ und aus $\mathfrak{T}$ zusammensetzt, also mit $\mathfrak{C}_{\mathfrak{r}} \mathfrak{T}$ zu bezeichnen ist. Sowohl durch $\mathfrak{T}$ als auch durch $\mathfrak{E}_{1} \mathfrak{T}$ können wir die zweite, zwischen $\gamma_{1}$ und $\gamma_{2}$ bestehende Perspektivität (bestimmt durch $L, L^{*} ; M, M^{*} ; N, N^{*}$ ) erzeugt denken; michin kann die ursprüngliche, infolge von $\mathscr{E}_{1}^{*} \mathscr{E}_{2}^{*}$ zwischen $\gamma_{1}$ und $\gamma_{2}$ bestehende Perspektivität hervorgerufen gedacht werden durch jede der beiden Collineationen mit Axen $\mathfrak{T} \mathfrak{T}$ und $\mathfrak{C}_{1} \mathfrak{T} \mathfrak{T}$, die beide $\bar{\gamma}_{1} \gamma_{2}$ als Axe der sich selbst entsprechenden Punkte und $\bar{C}_{1} \vec{C}_{2}$ als Axe der sich selbst entsprechenden Ebenen haben. Die Collineationen $\mathfrak{T} \mathfrak{\mathfrak { B }}$ und $\dot{\mathfrak{S}}_{1} \mathfrak{T} \mathfrak{H}$ führen beide $\Phi$ in $\Psi$ über und zwar so, dass den beiden Tangentialebenen $\varepsilon_{1}, \varepsilon_{2}$, die aus einer Geraden $g_{1}$ von $\gamma_{1}$ an $\Phi$ gehen, die beiden Tangentialebenen $\varepsilon_{1}^{\prime \prime}, \varepsilon_{2}^{\prime \prime}$, entsprechen, die aus der zu $g_{1}$ mit dem Centrum $C_{1}$ perspektivem Geraden $g_{2}$ von $\gamma_{2}$ an $\Psi$ gelegt werden können; es wird etwa in $\mathfrak{T} \mathfrak{G}$ der $\varepsilon_{1}$ die $\varepsilon_{1}^{\prime \prime}$ und der $\varepsilon_{2}$ die $\varepsilon_{2}^{\prime \prime}$, in der $\mathfrak{C}_{1} \mathfrak{T} \mathfrak{g}$ aber der $\varepsilon_{2}$ die $\varepsilon_{1}^{\prime \prime}$ und der $\varepsilon_{1}$ die $\varepsilon_{2}^{\prime \prime}$ zugeordnet sein. Hieraus erkennen wir, dass die durch $\mathbb{E}_{1}^{\circ} \mathbb{E}_{2}^{\prime \prime} z$ wischen den Berührungsebenen von $\Phi$ und $\boldsymbol{\Psi}$ hervorgerufene doppeldeutige Beziehung in zwei eindeutige, durch die Collineationen $\mathfrak{T} \mathfrak{T}$ und $\mathfrak{E}_{\mathfrak{I}} \mathfrak{T} \mathfrak{\mathfrak { E }}$ hervorgerufene zerfällt: Diese beiden Collineationen ordnen jeder Berührungsebene von $\Phi$ gerade die beiden ihr in $\mathscr{E}_{1}^{*} \mathscr{E}_{2}^{*}$ entsprechenden Berührungsebenen von $\Psi$ zu. - Jetzt nehmen wir eine beliebige Ebene $n$; ihr entsprechen in $\mathbb{F}_{1}^{*}$ zwei Ebenen und die diesen wieder in $\mathbb{E}_{2}^{*}$ entsprechenden Ebenen sind die vier durch $\mathscr{E}_{\mathfrak{s}}^{*} \mathfrak{E}_{2}^{*}$ der $n$ zugeordneten Ebenen. Der Tangentialkegel, dessen Ebenen

4) Vergl. Habilitationsschrift, Abschn. I, $\$ I, Nr. 2. 
$\boldsymbol{\Phi}$ in den Punkten des Schnittkreises $\left(\boldsymbol{\Phi} r_{1}\right)$ berühren, geht durch $\mathfrak{F}_{1}^{*} \mathfrak{F}_{2}^{*}$ in die beiden Tangentialkegel von $\Psi$ über, die ihm in $\mathfrak{T} \mathfrak{S}$ und $\mathfrak{S}_{\mathfrak{I}} \mathfrak{T} \mathfrak{S}$ entsprechen; diese beiden Kegel aber berühren die $\boldsymbol{\Phi}$ je in zwei Punkten und schneiden sie deshalb je in $z$ wei Ebenen, und die so erhaltenen vier Ebenen müssen die durch $\mathscr{F}_{1}^{*} \mathbb{E}_{2}^{*}$ der $n$ zugeordneten sein; denn auf $\mathbb{F}_{1}^{*} \mathfrak{F}_{2}^{*}$ geht die grundlegende Eigenschaft von $\mathfrak{F}_{1}^{*}$ und $\mathfrak{E}_{2}^{*}$ über, dass jede der einer Ebene ' entsprechenden Ebenen sich um eine Tangente von $\Phi$ dreht, wenn $\zeta$ dies tut. Jetzt erkennen wir aber sofort, dass die vier einer Ebene $\eta$ durch $\vec{F}_{1}^{*} \vec{F}_{2}^{*}$ zugeordneten Ebenen gerade die zweimal zwei Ebenen sind, die ihr in den beiden (5-Transformationen entsprechen, die von den Collineationen $\mathfrak{T} \mathfrak{S}$ und $\mathfrak{S}_{\mathfrak{1}} \mathfrak{T} \mathfrak{S}$ abhängen ${ }^{5}$ ). - Diese $(\mathfrak{E}$-Transformationen sind, wenn $\mathfrak{S}$ die fundamentale $\mathfrak{E}$-Transformation $\mathbb{E}_{0}$ bestimmt, $\mathfrak{T} \mathbb{E}_{0}$ und $\mathfrak{E}_{1} \mathfrak{I} \mathbb{E}_{0}$. Also haben wir gefunden:

Die Transformation $\mathbb{E}_{1}^{*} \mathbb{E}_{2}$, die durch Zusammensetzung zueier einfacher $\mathbb{E}$-Transformationen $\mathbb{F}_{1}^{*}, \mathbb{F}_{2}^{*}$ entstebt, zerfällt in zu'ei allgemeine $(\mathbb{E}$-Transformationen, die durch Zusammensetzung einer gewissen involutoriscben Centralcollineation $\mathfrak{T}$ und einer Collineation mit Axen $\mathfrak{S}_{1} \mathfrak{T}$ mit derselben fundamentalen $\mathbb{E}$-Transformation $\mathfrak{F}_{0}$ gebildet sind; wir deuten dies durch folgende Formel an:

$$
\mathbb{E}_{1}^{*} \mathbb{E}_{2}^{*}=\mathfrak{T} \mathbb{E}_{0}+\mathfrak{E}_{\mathrm{I}} \mathfrak{T} \mathbb{E}_{0}
$$

Die Hauptpunkte der sämtlicben bier vorkommenden Transjormationen liegen auf einer Geraden und die Hauptebenen gebören demselben Ebenenbüschel an.

(Hätten wir statt $\mathfrak{g}$ die andere Centralcollineation mit den Hauptelementen $P$ und $\pi$ genommen, die $\boldsymbol{\Phi}$ in $\Psi$ verwandelt, so hätten wir natürlich dieselben beiden $\&$-Transformationen erhalten, nur in der zweiten möglichen Zusammensetzung).

Unser Beweis versagt jedoch in dem Falle, dass $C_{1}$ in $\gamma_{2}$ und $C_{2}$ in $\gamma_{1}$ liegt; denn dann geht $\Phi$ durch $\mathfrak{F}_{\mathrm{I}}^{*} \mathbb{E}_{2}^{*}$ nicht in eine wirkliche Fläche II. Grades über, sondern in das Ebenenbündel $\left(C_{\mathrm{r}}\right)$. Dabei ist die Zuordnung zwischen den Berührungsebenen von $\Phi$ und den Ebenen des Bündels $\left(C_{1}\right)$ so, dass zwei Tangentialebenen von $\Phi$, die durch eine Gerade $g$ von $\gamma_{\mathrm{r}}$ laufen, die beiden Ebenen aus $\left(C_{\mathrm{I}}\right)$ entsprechen, die der Ebene $\left(C_{1} g\right)$ in $E_{2}^{*}$ zugeordnet sind. Ordnen wir die Schnittlinien der letzten beiden Ebenen mit $\gamma_{1}$ der $g \mathrm{zu}$, so erhalten wir in $\gamma_{\mathrm{I}}$ eine Geradenverwandtschaft, die in $\gamma_{1}$ durch die, vermöge $\mathbb{E}_{2}$ zwischen den Ebenen des Bündels $\left(C_{\mathrm{r}}\right)$ bestehende Beziehung eingeschnitten wird und das genaue ebene Analogon der Transformation $\mathbb{E}_{2}^{*}$ ist: Sie hat einen Grundkreis $\left(\Phi \gamma_{1}\right)$, ein Centrum $C_{2}$ und eine Hauptgerade $\overline{\gamma_{2}} \bar{\gamma}_{1}$; je zwei entsprechende Geraden schneiden sich auf $\overline{\gamma_{2} \gamma_{1}}$; insbesondere ist den aus einem Punkte von $\bar{\gamma}_{2} \gamma_{1}$ an $\left(\Phi \gamma_{1}\right)$ gehenden Tangenten die Verbindungslinie dieses Punktes mit $C_{2}$ zugeordnet; dreht sich eine Gerade um einen Punkt von $\left(\Phi{\gamma_{1}}_{1}\right)$, so tun die ihr entsprechenden Geraden desgleichen. Durch diese Eigenschaften ist die Verwandtschaft vollständig definiert, und wir können für sie genau die analoge Konstruktion ableiten wie für die Transformationen $\mathbb{E}^{*}$ in Raume. - Sei jetzt $\mathfrak{T}$ die eine der beiden involuto- 
rischen Centralcollineationen, die $\Phi$ in sich selbst und $\gamma_{1}$ in $\gamma_{2}$ überführen, $T$ ihr Centrum und $\tau$ ihre Hauptebene, so schneidet die durch sie erzeugte Beziehung zwischen den Berührungsebenen von $\Phi$ in $\gamma_{1}$ eine 3 -Transformation ${ }^{6}$ ) ein; diese aber hat wegen der besonderen Lageverhältnisse besondere, und zwar genau die oben angeführten Eigenschaften, ist also mit der obigen Geraden-Verwandtschaft identisch. Mithin erhalten wir die einer Berührungsebene $\varepsilon_{1}$ von $\Phi$ vermöge $\xi_{\mathrm{r}}^{*} \xi_{2}^{*}$ entsprechenden Ebenen auch dadurch, dass wir die beiden Ebenen $\varepsilon_{1}^{*}, \varepsilon_{2}^{*}$ nehmen, die der $\varepsilon_{1}$ und der ihr in der involutorischen Centralcollineation $\mathfrak{C}_{1}$ (mit den Hauptelementen $C_{\mathrm{t}}$ und $\gamma_{1}$ ) gepaarten $\varepsilon_{2}$ in $\mathfrak{T}$ entsprechen, und durch ihre Schnittlinien mit $\gamma_{1}$ die Ebenen aus $\left(C_{1}\right)$ legen, d. h. die Ebenen, die den $\varepsilon_{1}^{*}$ und $\varepsilon_{2}^{*}$ in $\varepsilon_{1}^{*}$ zugeordnet sind. Diese Ebenen entsprechen demnach der $\varepsilon_{1}$ (und der $\varepsilon_{2}$ ) in den Transformationen $\mathfrak{I}_{1}^{*}$ und $\mathfrak{E}_{1} \mathfrak{T} \mathfrak{E}_{1}^{*}$, und wir haben folgende Ergänzung zum letzten Satze:

Liegt das Centrum von $\mathbb{F}_{1}^{*}$ in der Hauptebene von $\mathbb{F}_{2}^{*}$ (und umgekehrt), so ist $\mathbb{E}_{\mathrm{o}} \equiv \mathbb{E}_{\mathrm{r}}^{*}$.

Ferner ist noch der besondere Fall anzumerken:

Es ist $\mathbb{E}^{*}=\mathbb{E}^{*} \mathbb{E}^{-1}=\mathbb{E}^{-1} \mathbb{E}=\mathrm{I}+\mathfrak{E}$,

wobei $I$ die Identität und $\mathbb{S}$ die involutoriscbe Centralcollineation bedeutet, die mit (5* die Hauptelemente gemeinsam bat.

\section{$\oint 2$.}

\section{Der Aufbau der Gruppe der (5-Transformationen mit Hilfe der einfachen \&-Transformationen.}

I. Bei gegebener Grundkugel $\Phi$ giebt es $\infty^{10}$ (5-Transformationen ${ }^{7}$ ), und darunter $\infty^{7}$, die sich wie $\mathfrak{T} \mathfrak{E}_{0}$ aus einer der $\infty^{3}$ involutorischen Centralcollineationen mit i. Bez. auf $\boldsymbol{\Phi}$ polaren Hauptelementen und aus einer der $\infty^{4} \mathrm{zu} \boldsymbol{\Phi}$ gehörigen fundamentalen (E-Transformationen zusammensetzen; sie konnen nicht alle als Teil einer zusammengesetzten Transformation wie $\mathscr{E}_{1}^{*} \xi_{2}^{*}$ auftreten, da es nur $\infty^{3}$ einfache $\mathbb{E}$-Transformationen und deshalb nur $\infty^{6}$ Kombinationen von je zwei solchen giebt. In der Tat können wir bei gegebener Transformation $\mathbb{E}_{0}$ die $\mathfrak{T}$ nicht beliebig wählen, wenn sich zwei einfache $\mathbb{E}$-Transformationen $\mathbb{F}_{5}$ und $\mathbb{E}_{2}$ der Art finden sollen, dass $\mathfrak{T} \mathbb{E}_{0}$ als Teil von $\mathscr{E}_{1}^{*} \mathbb{E}_{2}$ auftritt. Seien nämlich die Hauptelemente dieser Transformationen, wie früher, $P, \pi ; T, \tau ; C_{1}, \gamma_{1} ; C_{2}, \gamma_{2}$ und suchen wir $C_{1}, \gamma_{1}, C_{2}, \gamma_{2}$ aus den gegebenen $P, \pi ; T, \tau$ zu bestimmen, so sehen wir zunächst, wenn wir uns unserer früheren Figur erinnern, dass $\gamma_{2}$ durch $P$ und die Schnittgerade $\bar{\pi}$ und $C_{2}$ durch $\pi$ und die Gerade $\bar{P} \bar{T}$ gegeben sind; $\gamma_{1}$ ist dann die (durch $\overline{\tau \tau}$ laufende) Ebene des 
zweiten Kreises, den der jenige Kegel in $\Phi$ einschneidet, der aus $T$ den Schnittkreis $\left(\Phi \gamma_{2}\right)$ projiziert, und $C_{1}$ ist ihr auf $\overline{P T}$ liegender Pol. Aber $C_{1}$ muss die Bedingung erfüllen, dass der aus $C_{1}$ an $\Phi$ gehende Tangentialkegel die Ebene $\gamma_{2}$ in demselben Kegelschnitt schneidet wie die Fläche $\Psi$, in die $\Phi$ durch $\mathbb{E}$, übergeht.

Ist uns also $\mathbb{E}_{0}$ mit den Hauptelementen $P, \pi$ gegebenen, so werden wir eine involutorische Centralcollineation $\mathfrak{T}$ mit den Hauptelementen $T$, $\tau$ erst in besonderer Weise bestimmen müssen, wenn, kurz gesagt, $\mathfrak{T} \mathbb{E}_{0}$ ein Teil von $\mathbb{F}_{1}^{*} \mathbb{E}_{2}^{*}$ sein soll, und das geschieht folgendermassen: Wir wählen aus den $\infty^{2}$ vorhandenen Möglichkeiten $\mathscr{F}_{2}$ beliebig aus, indem wir für $\gamma_{2}$ eine beliebige durch $P$ laufende Ebene nehmen. Diese Ebene $\gamma_{2}$ schneidet die Fläche $\Psi$ in einem Kegelschnitt $\left(\Psi \gamma_{2}\right)$, der $\Phi$ in zwei (auf $\bar{\sigma} \gamma_{2}$ liegenden) Punkten berührt, und durch einen solchen Kegelschnitt gehen zwei Tangentialkegel von $\Phi$, deren Scheitel auf $\overline{P C_{2}}$ liegen; denn der duale Satz besagt, dass ein Kegel II. Grades, der $\Phi$ in zwei Punkten berührt, $\boldsymbol{\Phi}$ in zwei Kreisen schneidet. Jeden der beiden so erhaltenen Punkte von $\overrightarrow{P C}_{0}$ können wir als Centrum $C_{1}$ von $\mathscr{F}_{1}^{*}$ nehmen und haben dann für jede Wahl eine involutorische Centralcollineation $\mathfrak{T}$, die sich wie früher bestimmt. Mithin haben wir gefunden:

$Z u$ jeder fundamentalen E-Transformation $E_{\mathrm{o}}$ giebt es $\infty^{2}$ involutorische Centralcollineationen $\mathfrak{T}$, die die Grundkugel in sich selbst verwandeln und die Eigenschaft baben,

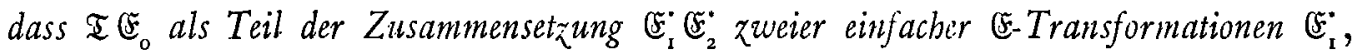
$\mathbb{E}_{2}^{*}$ dargestellt werden kann.

2. Aus zwei einfachen $\mathbb{E}$-Transformationen $\mathscr{E}_{1}^{*}, \mathbb{E}_{2}^{*}$ folgt die Transformation

$$
\mathbb{E}_{1}^{*} \mathbb{E}_{2}^{*}=\mathfrak{I E}_{0}+\mathbb{C}_{\mathrm{t}} \mathfrak{I} \mathbb{E}_{0},
$$

wobei $\mathfrak{T}$ eine involutorische Centralcollineation ist, die $\Phi$ in sich selbst und die Hauptelemente von $\mathbb{F}_{2}^{*}$ in die von $\mathscr{E}_{\mathrm{t}}^{*}$ überführt; deshalb ist

und ebenso auch

$$
\mathfrak{F}_{1}^{*}=\mathfrak{I F}_{2}^{*} \mathfrak{I}
$$

$$
\mathbb{E}_{1}=\mathfrak{T} \mathbb{E}_{2} \mathfrak{T},
$$

wenn $\widetilde{E}_{1}$, und $\mathfrak{E}_{2}$ die beiden involutorischen Centralcollineationen mit denselben Hauptelementen wie $\mathscr{E}_{\mathrm{I}}^{*}$ und $\mathbb{E}_{2}$ sind. Also ist auch

$$
\mathfrak{T} \mathbb{E}_{2}^{*} \mathfrak{T} \mathbb{E}_{2}^{\cdot}=\mathfrak{T} \mathbb{E}_{0}+\mathfrak{T} \mathbb{E}_{2} \mathfrak{T} \mathfrak{T} \mathbb{E}_{0}
$$

hieraus aber erkennen wir leicht, dass

$$
\mathbb{E}_{2} \mathfrak{T} \mathbb{E}_{2}=\mathbb{E}_{0}+\mathbb{E}_{2} \mathbb{E}_{0}
$$

ist, und das heisst, da wir ja $\mathscr{F}_{1}^{*}$ und $\mathscr{E}_{2}^{*}$, somit auch $\mathfrak{I}$ und $\mathbb{E}_{2}^{*}$ beliebig wählen können:

Ist $\mathfrak{F}^{*}$ eine einfache $\mathbb{E}$-Transformation mit der Grundkugel $\Phi$, $\mathbb{E}$ die involutorische Centralcollineation mit denselben Hauptelementen und $\mathfrak{T}$ eine andere $\Phi$ in sich selbst überfübrende involutorische Centralcollineation, so entstebt durch Anwendung von $\mathfrak{E}$ auf $\mathfrak{T}$ eine Transformation $\mathfrak{E} \mathfrak{T} \mathfrak{E}$, die in eine $z^{u}$ derselben Grundkugel $\mathbf{\Phi}$ gebörige fundamentale $\mathbb{E}$-Transformation $\mathbb{E}_{0}$ und in die Transformation $\mathbb{E} \mathbb{E}_{\mathrm{o}}$ zerfällt:

$$
\text { ⿷匚: }
$$


Wir fügen noch ergänzed hinzu :

Sind $C, \gamma$ die Haupteleminte von $\mathbb{F}^{*}$, ferner $T$, $\tau$ die von $T$ und $P, \pi$ die von $E_{0}$, so liegen immer $C, T, P$ in einer Geraden, wäbrend $\gamma, \tau, \pi$ sich in deren, $i$. Bez. auf $\Phi$ genommenen Polare schneiden; ausserdem liegt $C$ in $\pi$ und $P$ in $\gamma$.

Die folgenden beiden Spezialfälle sind anzumerken:

$$
\begin{aligned}
& \text { Ist } C \equiv T, \gamma \equiv \tau \text {; also } \mathbb{E} \equiv \mathfrak{I} \text {, so ist } \mathbb{E}^{*} \mathfrak{T} \mathbb{F}^{*}=\mathrm{I}+T \text {. } \\
& \text { Liegt } C \text { in } \tau \text { und } T \text { in } \gamma \text {, so ist } \mathbb{E}^{\mathfrak{T}} \mathfrak{E}=\mathfrak{T}+\mathfrak{C} \text {. }
\end{aligned}
$$

Nach dem letzten Satze des vorigen Absatzes aber können wir umgekehrt \& als beliebig gegeben annehmen und dazu auf $\infty^{2}$ Weisen $\mathfrak{T}$ und dann $\mathbb{E}^{\circ}$ bestimmen; das heisst:

Jede fundamentale ङ-Transformation erbält man auf doppelt unendlich viel Weisen durch Anwendung einer einfachen $\mathbb{E}$-Transformation auf eine involutorische Centralcolli. neation.

Da sich jede E-Transformation zusammensetzen lässt aus einer Anzahl von involutorischen Centralcollineationen der Grundkugel $\boldsymbol{\Phi}$ in sich und aus einer fundamentalen Transformation $\mathfrak{E}_{\mathrm{v}}$, folgt hieraus:

Die ganze Gruppe der (E-Transformationen kann man aus den in ibr befindlicben involutorischen Centralcollineationnen und einfachen \&-Transformationen aufbauten.

Es genügt dazu sogar schon eine einzige einfache (5-Transformation, da sich ja aus ihr nach $\ \mathrm{I}, 3$ alle anderen mit Hilfe der involutorischen Centralcollineationen ableiten lassen.

3. Als Ergänzungen führen wir noch die folgenden beiden Sütze - der Kürze halber ohne Beweis - auf :

Durch Anwendung einer cinfachen $\mathbb{E}$-Transformation $\mathfrak{E}^{*}$ auf eine fundamentale $\mathfrak{E}_{0}$, durch deren Centrum die Hauptebene von E läuft, entstebt eine vierdentige Transformation, die sich in vier Collineationen zerspaltet:

$$
\mathfrak{E}^{*} \mathbb{E}_{0} \mathbb{E}=\mathfrak{T}+\mathfrak{C} \mathfrak{T}+\mathfrak{T} \mathfrak{E}+\mathfrak{C} \mathfrak{T} \mathfrak{E}
$$

dabei sind $\mathfrak{I}$ und $\mathbb{E}$ zwei involutorische Centralcollineationen, und zwar ist $\mathfrak{E}$ diejenige, die dieselben Hauptelemente wie (5) bat.

Und :

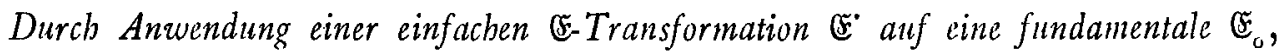
die mit ibr die Hauptelemente gerneinsam hat, entsteht eine vierdeutige Transformation

$$
\mathbb{E} \mathbb{E}_{0} \mathbb{E}=\mathbb{E}_{0}+\mathbb{E} \mathbb{E}_{0},
$$

wobei $\mathbb{5}$ wieder die involutorische Centralcollienation mit denselben Hauptelementen wie E* und [s ist.

Der Beweis dieses letzten Satzes beruht darauf, dass die Fläche $\Psi$, in die die Grundkugel $\Phi$, und die Fläche $\Psi^{\prime}$, in die das Ebenenbündel $(C)$ durch $\mathfrak{E}_{\mathrm{o}}$ und $\mathbb{E} \mathfrak{E}_{\mathrm{o}}$ verwandelt wird, in derselben Lagebeziehung zu einander stehen, wie die Flächen $X$ und $X^{\prime}$ des letzten Satzes von $\ \mathrm{I}, 2$, also einander in $\mathscr{E}^{*}$ entsprechen. Hierbei ergiebt sich nebenbei; da durch $\Psi^{\prime}$ die Fläche $\Psi$ und damit wieder $\mathbb{E}_{0}$ und $\mathbb{E} \mathbb{E}_{0}$ bestimmt sind, der Satz: 
Bei gegebener Grundkugel $\Phi$ sind durch eine, iłor umscbriebene Fläcbe II. Grades $\Psi^{\prime}$ zwei fundamentale (E-Transformationen $\mathbb{E}_{0}$ und $\mathbb{C}_{0}$ bestimmt, in denen $\Psi^{\prime}$ von den Ebenen umbïllt wird, deren entsprechende durch das Centrum der Transformationen laufen.

Statt $\Psi^{\prime}$ brauchen auch nur die Hauptelemente und eine Berührungsebene von $\Psi^{\prime}$ gegeben zu sein.

\section{$\ 3$.}

\section{Die Bedeutung der einfachen \&.Transformationen für die Berührungstransformationen der Kreise einer Ebene.}

I. Betrachten wir jetzt die zu einer Grundkugel $\Phi$ gehörigen E-Transformationen und projizieren die Transformationen, die sie in $\Phi$ einschneiden, aus einem nicht auf I gelegenen Punkte $S$ auf seine i. Bezug auf $\Phi$ genommenen Polarebene $\sigma$, so erhalten wir ${ }^{8}$ ) in $\sigma$ die Berührungstransformationen der Kreise derjenigen Massbestimmung, deren absoluter Kegelschnitt der Schnittkreis von $\sigma$ mit $\Phi$ ist. Insbesondere liefert uns die einfache $\mathbb{E}-$ Transformation $\mathbb{E}_{0}^{*}$, deren Hauptelemente $S$ und $\sigma$ sind, nach $\$ I, I das absolute Polarsystem von $\sigma$.

Die Ergebnisse von $\ 2,2 u .3$ führen uns genau auf die Sätze, die in I, $\S$ I, 3 abgeleitet worden sind. Ist nämlich $\mathfrak{T}$ eine der zur Grundkugel $\boldsymbol{\Phi}$ gehörigen involutorischen Centralcollineationen und geht ihre Hauptebene durch $S$, so ist $\mathfrak{E}_{0} \mathfrak{T} \mathfrak{E}_{0}^{*}=\mathfrak{T}+\mathfrak{S} \mathfrak{T}$, wobei $\subseteq$ die involutorische Centralcollineation mit den Hauptelementen $S$, $\sigma$ ist; $\mathfrak{T}$ und $\subseteq \mathfrak{T}$ liefern in $\approx$ dieselbe nichteuklidische Spiegelung; eine solche wird also durch Anwendung des absoluten Polarsystems nicht geändert. Geht die Hauptebene von $\mathfrak{T}$ nicht durch $S$, so ist $\mathbb{E}_{0}^{*} \mathfrak{T} \mathbb{E}_{0}^{*}=\mathbb{E}_{0}+\mathfrak{S} \mathbb{E}_{0}$, wobei $\mathbb{E}_{0}$ eine fundamentale $\mathbb{E}-$ Transformation ist, deren Hauptebene durch $S$ läuft; es liefern nun in $\sigma: \mathfrak{T}$ eine nichteuklidische Inversion, $\mathfrak{E}_{0}$ und $\mathbb{S}_{\mathbb{E}_{0}}$ aber dieselbe 8 -Transformation; also geht die erstere durch Anwendung des absoluten Polarsystems von $\sigma$ gerade in die letztere über. Ferner sei $\mathbb{E}_{0}$ eine zur Grundkugel $\Phi$ gehörige fundamentale $\mathbb{E}$-Transformation. Geht die Hauptebene von $\mathfrak{E}_{0}$ durch $S$, so wird $\mathfrak{E}_{0}^{*} \mathfrak{E}_{0}=\mathfrak{I}+\mathfrak{S} \mathfrak{T}+\mathfrak{I} \mathfrak{S}+\mathfrak{S} \mathfrak{T} \subseteq$, und das bedeutet, dass in $\sigma$ jede 8 -Transformation durch Anwendung des absoluten Polarsystems in eine Inversion verwandelt wird. Hat endlich $\mathbb{E}_{0} S$ und $\sigma$ zu Hauptelementen, so folgt aus der Formel $\mathscr{E}_{0}^{*} \mathbb{E}_{0} \mathfrak{E}_{0}^{*}=\mathscr{E}_{0}+\subseteq \mathfrak{S}_{0}$, dass in $\sigma$ jede Dilatation bei Anwendung des absoluten Polarsystems ungeändert bleibt.

2. Die einfachen \&-Transformationen, deren Hauptebenen durch das Projektionscentrum $S$ laufen, führen in o zu Transformationen der Geraden, die ihnen genau analoge Eigenschaften haben und Spezialfälle der 8 -Transformationen sind; sie sollen deshalb " einfache 3-Transformationen » heissen ${ }^{9}$ ). In jeder 3-Transformation entspricht

8) I, $\ I, 2$.

9) Wir haben eine solche einfache 3 -Transformation schon vorübergehend in $\$ \mathrm{I}, 4$ benützt. 
dem absoluten Kegelschnitt ein ihn doppelt berührender Kegelschnitt, der «Fluchtkreis »; bei den einfachen 8-Transformationen entartet der Fluchtkreis in einen Punkt, nämlich in den Hauptpunkt. Nehmen wir zwei einfache $\mathbb{E}$-Transformationen $\mathbb{E}_{1}^{*}$ und $\mathbb{E}_{2}^{*}$, deren Hauptebenen durch $S$ laufen, und bilden nach $\int \mathrm{I}, 4 \mathfrak{F}_{1}^{*} \mathfrak{E}_{2}^{*}=\mathfrak{T} \mathbb{E}_{0}+\mathfrak{E}_{1} \mathfrak{T} \mathbb{E}_{0}$, so gehören die Hauptebenen von $\mathfrak{I}$ und $\mathfrak{E}_{\mathrm{o}}$ dem Ebenenbüschel an, der durch die Hauptebenen von $\mathbb{E}_{1}^{*}$ und $\mathfrak{F}_{2}^{*}$ bestimmt ist; $\mathfrak{T}$ liefert in $\sigma$ wiederum eine involutorische Centralcollineation oder Spiegelung und $\mathbb{E}_{0}$ eine allgemeine 3-Transformation; also können wir das Ergebnis von $\ \mathrm{I}, 4$ auf die einfachen 8 -Transformationen übertragen. Genau dasselbe gilt von den Ergebnissen in $\ 2$, I und 2; insbesondere haben wir:

Jede nichteuklidische 3-Transformation erbält man auf einfach unendlich viele $W$ eisen durch Anwendung einer einfachen 3-Transformation auf eine Spiegelung.

3. Eine nichteuklidische Inversion $\mathfrak{j}$ der Ebene $\sigma$ entsteht in der früher angegebenen Weise aus einer involutorischen Centralcollineation $\mathfrak{T}$, die die Kugel $\Phi$ in sich selbst überführt; sind $\mathfrak{I}$ und $\tau$ die Hauptelemente von $\mathfrak{T}$, so ist die Schnittgerade $c \equiv \overline{\tau \tau}$ die "Axe " $\left.{ }^{\text {Io }}\right)$ der Inversion $\mathrm{j}$. Die Ebene $\gamma \equiv(S, c)$ bestimmt eine einfache E-Transformation E , mit den Hauptelementen $C$ und $\gamma$, und diese liefert in $\sigma$ die durch $c$ als Hauptgerade bestimmte einfache 3 -Transformation 3 . Wenden wir jetzt $\mathbb{E}^{*}$ auf $\mathfrak{T}$ an, so erhalten wir nach dem Hauptsatze von $\ 2,2$

$$
\mathbb{E} \mathfrak{T} \mathbb{E}=\mathbb{E}_{\mathrm{o}}+\mathfrak{E} \mathbb{E}_{\mathrm{o}},
$$

worin $\mathbb{E}$ die involutorische Centralcollineation mit den Hauptelementen $\mathbb{E}$ und $\gamma$ und $\mathfrak{E}_{0}$ eine fundamentale $\mathbb{E}$-Transformation mit den Hauptelementen $P \equiv(\overline{C T}, \gamma)$ und $\pi \equiv(c, C)$ ist. Nach unserer Voraussetzung über $\mathscr{F}^{*}$ aber ist $S \equiv(C T, \gamma)$ und $\sigma \equiv(c, C)$, also $P \equiv S$ und $\pi \equiv \sigma$. Deshalb geht $\left.{ }^{\text {II }}\right)$ bei der Projektion aus $S \mathbb{F}_{\mathrm{o}}$ in eine Dilatation $\mathfrak{D}$ und $\mathfrak{C}$ in eine Spiegelung $\{$ der nichteuklidischen Ebene $\sigma$ über, und wir erhalten in dieser durch Anwendung von $3^{\circ}$ auf $\mathrm{j}$ :

das heisst:

$$
8^{*} \mathrm{i} 8^{*}=\mathfrak{D}+\mathfrak{D}
$$

Wendet man in einer Ebene mit nichteuklidischer Massbestinmung auf eine Inversion i die einfache $8^{-T r a n s f o r m a t i o n ~} 8^{\circ}$ an, deren Hauptgerade die Axe $c$ von i ist, so zerfällt die Transformation $8^{*} \mathrm{i} 8^{*}$ in zwei Transformationen, von denen die eine eine $D i$ latation $\mathfrak{D}$ ist und die andere sich aus der nichteuklidischen Spiegelung $\lceil$ an $c$ und aus $D$ zusammensetzt, wie es die obige Gleichung andeutet.

Ist uns umgekehrt in $\sigma$ eine Dilatation gegeben, so führt diese zu einer fundamentalen $\mathbb{E}$-Transformation $\mathbb{E}_{\text {。 }}$ zurück, deren Hauptelemente $P \equiv S$ und $\pi \equiv \sigma$ sind; nach $\ 2$, I und 2 können wir zu jeder einfachen Transformation $\mathbb{E}$, deren Hauptebene $\gamma$ durch $P$ läuft, in doppelter Weise eine involutorische Centralcollineation $T$ bestimmen, derart, dass $\mathfrak{E}_{0}$ als Teil von $\mathbb{E}^{*} \mathfrak{T} \mathbb{E}^{*}$ erscheint. Daraus folgt:

In einer Ebene mit nichteuklidischer Massbestimmung erbält man jede Dilatation auf $\infty^{2}$ Weisen durch Anwendung einer einfachen 3-Transformation auf eine Inversion.

I o) Habilitationsschrift, I. Abschn., $\$ 2,3$.

II) I, $\mathbb{I}, 2$.

Resd. Circ. Matem. Palermo, t. XXVI (2 ${ }^{\circ}$ sem. 1908), - Stampato il 3 giugno 1908. 
4. Ist in den Betrachtungen des vorigen Absatzes die involutorische Centralcollineation $\mathfrak{T}$ so bescaffen, dass der Ebene $\sigma$ durch $\mathfrak{T}$ gerade die Ebene $\gamma$ zugeordnet wird, die die Schnittlinie $c \equiv \overline{\sigma \tau}$ mit $S$ verbindet, so entspricht in 9 dem absoluten Kegelschnitt $(\sigma \Phi)$ die Gerade $c$, d. h. $\dot{\jmath}$ ist eine solche Inversion $\dddot{j}^{*}$, deren Fluchtkreis entartet und mit ihrer Axe zusammenfält. Wir nennen eine solche Inversion eine " einfache Inversion ", weil die ihr im absoluten Polarsystem der Ebene $\sigma$ entsprechende 3-Transformation einen Fluchtkreis hat, der in ihren Hauptpunkt entartet, und deshalb eine einfache 3 -Transformation ist ${ }^{\mathbf{1}}$ ). Nehmen wit jetzt wieder die einfache (E-Transformation $\mathbb{E}^{*}$, deren Hauptebene $\gamma$ und deren Hauptpunkt der Pol von $\gamma$ i. Bez. auf die Grundkugel ist, so hat die Transformation $\mathfrak{E}^{*} \mathfrak{T} \mathfrak{E}=\mathfrak{E}_{0}+\mathfrak{E} \mathfrak{E}_{\mathrm{o}}$ die Besonderheit, dass sie die Berührungsebenen der Grundkugel $\Phi$ in die Ebenen überführt, die durch das Projektionscentrum $S$ laufen; denn den Berührungsebenen von $\Phi$ entsprechen in $\mathfrak{E}$ die Ebenen des Bündels $(C)$, diesen aber in $\mathfrak{T}$ - wegen der vorausgesetzten Besonderheit von $\mathfrak{T}$ - die Ebenen des Bündels $(S)$, und diese werden, da $S$ in $\gamma$ liegt, wiederum durch $\mathfrak{F}^{*}$ nur untereinander vertauscht. Darum ist in diesem Falle $\mathfrak{E}_{\mathrm{o}}$ die einfache Transformation $\mathscr{E}_{0}^{*}$, deren Hauptelemente $S$ und $\sigma$ sind, und es liefert, wenn wir zur Geometrie in der Ebene $\sigma$ übergehen, die Zusammensetzung $3^{*} i^{\prime \prime} 3^{\circ}$ nicht eine eigentliche Dilatation $\mathfrak{D}$, sondern das absolute Polarsystem.

Wendet man also in einer Ebene mit nichteuklidischer Massbestimmung auf eine einfache Inversion $\ddot{\mathrm{j}}$ die einfache 3 -Transformation $3 \overrightarrow{3}$ an, deren Hauptgerade die Axe $c$ von $\ddot{j}^{*}$ ist, so zerfällt die Transformation $3^{\circ} \mathrm{j}^{*} 3^{*}$ in zwei Transformationen, von denen die eine das absolute Polarsystem ist und die andere sich aus diesem und der (nichteuklidischen) Spiegelung an c zusammensetzt.

5. Da durch das absolute Polarsystem die Spiegelungen und Dilatationen nicht geändert werden, die Inversionen und 3-Transformationen aber mit einander - und insbesondere die einfachen Inversionen mit den einfachen 3-Transformationen - vertauscht werden, können wir jedem Satze der letzten beiden Absätze einen ihm polaren Satz zur Seite stellen. Ferner gilt ebenso, wie in $\ \mathrm{I}, 3$ für die einfachen \&-Transformationen, auch für die einfachen 3 -Transformationen und für die einfachen Inversionen, dass sie alle mit Hilfe der Spiegelungen aus einer einzigen von ibnen hervorgehen. $\mathrm{Da}$ endlich alle Berührungstransformationen der Kreise einer Ebene sich aus den Spiegelungen, Dilatationen, Inversionen und 3-Transformationen zusammensetzen lassen, so können wir als Endergebnis den Satz aussprechen:

In einer Ebene mit nicbteuklidischer Massbestimmung kann man alle Berïbrungstransformationen der Kreise berstellen aus den Spiegelungen, dem absoluten Polarsystem und entweder einer einfacben Inversion oder einer einfachen 3-Transformation - bezw. aus den Spiegehungen, einer einfachen Inversion und einer einfacben 3-Transformation.

Braunschweig, Dezember I907.

W. LUDWIG.

ra) Die einfachen Inversionen sind die $L$-Transformationen, die Herr H. LiebmanN auf anderem Wege gefunden hat; siehe Berichte der Leipziger Gesellschaft der Wissenschaften, Bd. LIV (I902), Seite 244, ff. 